\title{
Concepts are not represented by conscious imagery
}

\author{
Diane Pecher and Saskia van Dantzig \\ Erasmus University Rotterdam, Rotterdam, The Netherlands \\ AND \\ HENDRIK N. J. SCHIFFERSTEIN \\ Delft University of Technology, Delft, The Netherlands
}

\begin{abstract}
According to theories of grounded cognition, conceptual representation and perception share processing mechanisms. We investigated whether this overlap is due to conscious perceptual imagery. Participants filled out questionnaires to assess the vividness of their imagery (Questionnaire on Mental Imagery) and the extent to which their imagery was object oriented and spatially oriented (Object-Spatial Imagery Questionnaire), and they performed a mental rotation task. One week later, they performed a verbal property verification task. In this task, involvement of modality-specific systems is evidenced by the modality-switch effect, the finding that performance on a target trial (e.g., apple-green) is better after a same-modality trial (e.g., diamond-sparkle) than after a different-modality trial (e.g., airplane-noisy). Results showed a modality-switch effect, but there was no systematic relation between imagery scores and modality switch. We conclude that conscious mental imagery is not fundamental to conceptual representation.
\end{abstract}

How are objects represented in people's minds? Recent evidence suggests that mental representation shares mechanisms with perception and action. In other words, sensorymotor systems support mental concepts. In the present study, we investigated whether conscious perceptual imagery is the mechanism that underlies mental representation.

Several theories have proposed that concepts are grounded in sensory-motor processing (Barsalou, 1999, 2008; Glenberg, 1997; Pulvermüller, 1999). Object concepts are learned through recurrent experiences of perceiving and interacting with the object. These experiences are captured by modality-specific sensory-motor systems and integrated via hierarchical association areas (Barsalou, Simmons, Barbey, \& Wilson, 2003). Representation is achieved by simulators that reactivate aspects of experience in a top-down fashion from higher level association areas back to the modality-specific sensory-motor systems. It is important to note that the actual representation is achieved by the sensory-motor systems and not by the higher level association areas, and that this aspect of the theory is essentially different from a symbolic account of cognition, which would assume that abstract representations suffice for concepts. A middle position was proposed by Mahon and Caramazza (2008), who argued that sensory-motor systems enrich representations, but that concepts are at least partially abstract.

Evidence for grounded representations comes from both behavioral and neuroimaging studies (Goldberg, Perfetti,
\& Schneider, 2006; Martin, Haxby, Lalonde, Wiggs, \& Ungerleider, 1995; Pecher, Zeelenberg, \& Barsalou, 2003, 2004; Solomon \& Barsalou, 2001; Stanfield \& Zwaan, 2001; but see Mahon \& Caramazza, 2008). In the present article, we focus on a particular body of evidence that supports the idea that modality-specific sensory-motor systems are used to represent concepts. Pecher et al. (2003) demonstrated that, just as in perceptual tasks (Spence, Nicholls, \& Driver, 2001), switching between sensory modalities in conceptual judgments incurred a processing cost. Concept names were presented with a property name from a specific modality in a property verification task. Responses were faster and more accurate if a target trial (e.g., apple-green) was preceded by a context trial from the same modality (e.g., diamond-sparkling) than if it was preceded by a context trial from a different modality (e.g., airplane-noisy). The modality-switch effect has been replicated (Marques, 2006) and extended to switching between affective and perceptual representations (Vermeulen, Niedenthal, \& Luminet, 2007). Importantly, the modality-switch effect was also found when the conceptproperty trial was preceded by an actual perceptual trial (e.g., a light flash or beep) (van Dantzig, Pecher, Zeelenberg, \& Barsalou, 2008). Further, property verification is affected by perceptual memory load (Vermeulen, Corneille, \& Niedenthal, 2008). These studies strongly suggest that conceptual knowledge is grounded in modalityspecific perceptual systems.

D. Pecher, pecher@fsw.eur.nl 
An important assumption in the explanation of modality-switch effects in conceptual tasks is that representations are flexible and context dependent. Different modality-specific sensory-motor systems may contribute to a representation in various degrees, depending on their relevance for the task. To verify that an apple can be green, the visual sensory system is activated most strongly, in order to create a predominantly visual representation of "apple." To verify that an apple can be tart, the gustatory system contributes most to the representation. If consecutive trials contain properties from different modalities, attention has to be switched from one modality-specific system to another, causing a modality-switch effect. The studies by van Dantzig et al. (2008) and Vermeulen et al. (2008) indicate the involvement of modality-specific sensory systems. Consistent with this claim is the finding in fMRI studies of activation of modality-specific brain areas while participants performed property verification or similar conceptual tasks (Goldberg et al., 2006; Martin et al., 1995).

Some researchers have suggested that the involvement of sensory-motor systems in cognitive tasks is similar to mental imagery. Mental imagery and perception have been shown to share modality-specific mechanisms (Rouw, Kosslyn, \& Hamel, 1997), and both concept representation and imagery activate brain regions that overlap with areas activated during perceptual processing (Borst \& Kosslyn, 2008). On the other hand, the processes involved in imagery are quite different from those involved in representing conceptual knowledge (Barsalou, 1999, 2009). An important difference is the degree of effortful conscious control. The act of scanning, reorganizing, or rotating a mental image requires someone to actively maintain a mental image and perform operations on that image, whereas the act of retrieving conceptual knowledge happens largely outside one's conscious control. Since perceptual simulation is assumed to underlie many cognitive processes, most of it happens without conscious awareness or control (but see Vermeulen et al., 2008).

Another difference is that imagery is an ability or tendency that varies widely among individuals (Blajenkova, Kozhevnikov, \& Motes, 2006; Burton \& Fogarty, 2003; Kosslyn, Brunn, Cave, \& Wallach, 1984), whereas representing conceptual knowledge is performed easily by anyone with normal cognitive abilities. Thus, concepts may rely on automatic activation of modality-specific systems independent of an individual's ability or tendency to use mental imagery. The role of sensory-motor systems might be fundamental for conceptual knowledge, as proposed by theories of grounded cognition. On the other hand, it is possible that only some people use sensory-motor simulations, whereas others use different, more symbolic mechanisms. Thus, data that have been presented as support for sensory-motor simulations might actually consist of a mixture of sensory-motor and null effects that, when averaged, might still present small sensory-motor effects. Empirical evidence on this issue is scarce and mixed. Some studies suggest that there is no relation between imagery and the perceptual processing involved in language comprehension (Stanfield \& Zwaan, 2001), whereas other studies show that scores on imagery tests are related to cognitive tasks, especially when the same operations are involved (Blajenkova et al., 2006; Kozhevnikov, Kosslyn, \& Shephard, 2005; Laws, 2002; Marks, 1973).

Interestingly, Solomon and Barsalou (2004) demonstrated that participants behaved similarly under visual imagery and neutral instructions in a property verification task. Orthogonal to instruction, they manipulated whether the false trials contained associated conceptproperty pairs (e.g., owl-tree). When only the true trials were associated, participants could use superficial processing based on word associations. When both true and false trials were associated, however, participants had to use deeper processing, and, in that case, perceptual variables affected their performance. Imagery participants as well as neutral participants were strongly affected by the presence or absence of associations. This could mean that, even when instructed, participants were not using imagery to perform the task if they could rely on other information, such as word association. Therefore, the question remains whether the imagery instruction always induced imagery behavior. Although this study provides strong and appealing evidence for perceptual simulation, it does not necessarily show that simulation and imagery are similar.

In the present study, we investigated the relation between imagery ability and the conceptual modality-switch effect. We used several measures to assess imagery ability: the Questionnaire on Mental Imagery (QMI; Betts, 1909; Sheehan, 1967), which measures vividness of imagery in different modalities, the Object-Spatial Imagery Questionnaire (OSIQ; Blajenkova et al., 2006), which measures tendency and experience to use object-oriented and spatial visual imagery, and a mental rotation task that measures actual performance rather than self-reported ability. This task requires knowledge retrieval (visual letters) and speeded responses and, thus, shares components with property verification. Finally, participants performed a property verification task in which modality switch was manipulated as in the Pecher et al. (2003) study. If the modality switch effect is driven mainly by those participants who use conscious imagery, a higher imagery score should predict a larger modality-switch effect (Laws, 2002). People with very vivid imagery focus more attention on modality-specific information and, therefore, should show larger costs associated to switching attention to a different modality. Preference for object imagery (as measured by the OSIQ) should also be related to the modality-switch effect. High object imagers focus more on perceptual object properties (Kozhevnikov et al., 2005) and, therefore, would suffer more from switching modalities than would low object imagers. Finally, if the modality-switch effect is due to conscious imagery, we would expect the effect to be correlated with mental rotation, which is another performance measure of the use of imagery. 
Table 1

Descriptive Statistics

\begin{tabular}{|c|c|c|c|c|}
\hline Task & $M$ & $S E$ & Range & $\alpha$ \\
\hline \multicolumn{5}{|c|}{ QMI: (1-7 scale, $1=$ most vivid, 5 items per subscale) } \\
\hline Auditory & 2.51 & 0.13 & $1.00-5.80$ & .81 \\
\hline Tactile & 3.14 & 0.14 & $1.00-5.00$ & .77 \\
\hline Visual & 2.21 & 0.12 & $1.20-5.40$ & .71 \\
\hline Gustatory & 2.92 & 0.15 & $1.00-6.00$ & .77 \\
\hline Olfactory & 2.89 & 0.15 & $1.00-6.20$ & .81 \\
\hline Kinaesthetic & 3.03 & 0.14 & $1.20-6.60$ & .83 \\
\hline Organic & 2.47 & 0.11 & $1.00-6.00$ & .73 \\
\hline \multicolumn{5}{|c|}{ OSIQ: (1-5 scale, $1=$ least agree, 15 items per subscale) } \\
\hline Object oriented & 3.55 & 0.06 & $2.27-4.33$ & .69 \\
\hline Spatial & 2.70 & 0.09 & $1.47-4.20$ & .84 \\
\hline \multicolumn{5}{|c|}{ Rotation (msec/deg, 48 items) } \\
\hline Slope & 2.52 & 0.32 & $-3.17-12.29$ & $.81^{\mathrm{a}}$ \\
\hline \multicolumn{5}{|c|}{ Property verification times switch effect ( $\mathrm{msec}, 40$ items per modality) } \\
\hline Auditory & 39 & 16 & $-231-356$ & $.89^{b}$ \\
\hline Tactile & 16 & 17 & $-274-409$ & .89 \\
\hline Visual & 12 & 16 & $-242-487$ & .87 \\
\hline
\end{tabular}

Property verification accuracy switch effect (proportion)

\begin{tabular}{lllll} 
Auditory & .02 & .01 & $-.21-19$ & .39 \\
Tactile & .01 & .01 & $-.27-.23$ & .32 \\
Visual & .02 & .01 & $-.21-.26$ & .32 \\
\hline
\end{tabular}

Note-QMI, Questionnaire on Mental Imagery; OSIQ, Object-Spatial Imagery Questionnaire. ${ }^{a}$ Mean RTs per rotation angle were used to compute Cronbach's alpha. 'bFor property verification, the mean Cronbach's Alphas are reported (averaged over the no-switch and switch conditions for different counterbalanced versions).

\section{METHOD}

\section{Participants}

Sixty students at the Erasmus University Rotterdam participated for course credit.

\section{Stimuli}

To assess vividness of imagery, we used a modified Dutch translation of the shortened version of Betts's QMI (Betts, 1909; Sheehan, 1967), ${ }^{1}$ which assesses imagery ability for seven sensory modalities: visual, auditory, cutaneous (tactile), kinaesthetic, gustatory, olfactory, and organic (visceral), with five items for each modality. Participants were asked to think about experiencing the item in that modality and to carefully consider the image that came to mind. They rated the image on a scale of $1-7$, where $1=$ perfectly clear and vivid as the actual experience, and $7=$ no image at all, you only know that you are thinking of the object (descriptions for all five intermediate points were also provided).

Object and spatial visual imagery was assessed by a Dutch translation of the OSIQ (Blajenkova et al., 2006). This questionnaire has 30 items, 15 for each subscale (spatial and object). Each item is a statement such as "Architecture interests me more than painting" (spatial subscale) or "I can close my eyes and easily picture a scene that I have experienced" (object subscale). Participants indicate their agreement on a scale from $1=$ totally disagree to $5=$ totally agree. The test-retest reliability (after a week interval) is high (.95 for spatial and .81 for object; Blajenkova et al., 2006), indicating that it measures a stable trait.

In the mental rotation task, participants had to decide whether the capital letters "R" and "F" were displayed correctly or as a mirror image. Combining the 4 stimuli in 12 different rotation angles $\left(0^{\circ}\right.$, $\left.30^{\circ}, 60^{\circ}, \ldots, 330^{\circ}\right)$ resulted in 48 stimuli. A practice set consisted of six pictures displaying a correct or mirror image of the capital letter "G" in different angles.

For property verification, 240 concept-property pairs were used as experimental items, 80 each from the visual, auditory, and tactile modality (van Dantzig \& Pecher, 2009; van Dantzig et al., 2008). All properties were true for the concept. The pairs were combined (e.g., "A tomato is red."). Half were used as context items, and half were used as target items. For each modality, 20 target items were preceded by a same-modality context item and 20 were preceded by a different-modality context item (10 each from the two different modalities). Four versions of the stimulus list were created to counterbalance items over conditions. Across lists, each item was presented twice in the same-modality condition and twice in the different-modality condition. Each participant received only one list, with 15 participants per list.

In addition, 220 false and 60 true filler items were created. Following Pecher et al. (2003) and Solomon and Barsalou (2004), 70 false fillers contained words that were associatively related (e.g., "an onion can cry," "a book can read") to promote deep semantic processing. The remaining 150 false fillers contained visual, auditory, or tactile properties. The 60 true fillers had similar types of properties as the associated false properties. For practice, 20 true and 20 false filler items were presented.

\section{Procedure}

In order to minimize awareness of the goal of the study. participants were tested in two sessions, separated by a week, as part of a larger set of experiments. During the first session, the mental rotation task, the QMI, and the OSIQ were administered. In the mental rotation task, participants were instructed to decide as quickly as possible whether a letter was displayed correctly or as a mirror image. On each trial, a letter was presented on the computer screen; participants indicated "correct" by pressing the " $\mathrm{M}$ " key or "mirror image" by pressing the " $Z$ " key. Following an incorrect response, feedback ("fout"-error) was presented for 1,000 msec. After a 250 -msec interval, the next trial started. First, 6 practice trials were presented, followed by 48 experimental trials. The order of stimuli was randomized for each participant.

Both questionnaires were also presented on the computer screen, one item at a time. All response options were presented below the item in individual boxes containing the value of the option (e.g., totally agree), and participants clicked on the box of their choice.

A week later, participants performed the property verification task. A trial started with a fixation mark $(*)$ presented for $1,000 \mathrm{msec}$. Subsequently, the concept-property pair was presented until the participant responded or 3,500 msec had elapsed. Participants pressed the "M" key to indicate "true" or the "Z" key to indicate "false." Feedback was provided for 1,500 msec if the participant had made an error ("fout"-error) or did not respond

Table 2

Property Verification Reaction Times (RTs, in Milliseconds) and Accuracy

\begin{tabular}{|c|c|c|c|c|c|c|c|c|c|c|c|c|}
\hline \multirow[b]{4}{*}{ Condition } & \multicolumn{12}{|c|}{ Modality of Target Trial } \\
\hline & \multicolumn{4}{|c|}{ Auditory } & \multicolumn{4}{|c|}{ Tactile } & \multicolumn{4}{|c|}{ Visual } \\
\hline & \multicolumn{2}{|c|}{ RT } & \multicolumn{2}{|c|}{ Accuracy } & \multicolumn{2}{|c|}{ RT } & \multicolumn{2}{|c|}{ Accuracy } & \multicolumn{2}{|c|}{ RT } & \multicolumn{2}{|c|}{ Accuracy } \\
\hline & $M$ & $\overline{S E}$ & $M$ & $\overline{S E}$ & $M$ & $\overline{S E}$ & $\bar{M}$ & $\overline{S E}$ & $M$ & $\overline{S E}$ & $M$ & $S E$ \\
\hline No sw & 1,069 & 25 & .94 & .01 & 1,106 & 24 & .91 & .01 & 1,082 & 24 & .93 & .01 \\
\hline Switch & 1,108 & 26 & .92 & .01 & 1,122 & 28 & .90 & .01 & 1,094 & 23 & .91 & .01 \\
\hline
\end{tabular}


Table 3

\begin{tabular}{ccc}
$\begin{array}{c}\text { Correlations Among Scores for the Overall Modality-Switch } \\
\text { Effects for QMI, OSIQ, and Rotation Slopes }\end{array}$ \\
\hline & \multicolumn{2}{c}{ Modality Switch Effect } \\
\cline { 2 - 3 } & RT & Accuracy \\
\hline QMI & .14 & -.02 \\
OSIC scales & & \\
Object & -.15 & .16 \\
Spatial & -.02 & -.19 \\
Rotation slope & -.02 & .19 \\
\hline
\end{tabular}

Note- QMI, Questionnaire on Mental Imagery; OSIQ, Object-Spatial Imagery Questionnaire.

within 3,500 msec ("te laat"-too late). After $250 \mathrm{msec}$, the next trial started. Following every 130 trials, there was a self-paced break, during which accuracy statistics of the preceding block of trials were presented. If accuracy was below $85 \%$, participants were urged to be more accurate. If it was above $95 \%$, participants were complimented on their score. Items were presented in a different random order for each participant, with the exception that target items always followed their context item.

\section{RESULTS}

For each participant, we calculated the mean ratings on each of the modality subscales of the QMI and the object and spatial subscales of the OSIQ (after reverse coding of one negatively formulated item). Correct mental rotation reaction times (RTs) that fell within $3 S D$ s from the participant's mean were used to compute the slope of the rotation function (using the shortest of the clockwise and counterclockwise rotation angles for each stimulus). Descriptive statistics are listed in Table 1.

In the property verification task, mean RTs were based on trials for which the responses were correct on the tar- get trial as well as on the context trial and that fell within $3 S D$ s of the participant's condition mean, resulting in $81 \%$ valid RTs. Mean RTs for switch and no-switch trials for each modality were computed for each participant and were then averaged across participants. Mean RTs and accuracy are presented in Table 2 . Two 2 (switch) $\times 3$ (target modality) ANOVAs on the RTs and error rates showed that the effect of modality switch was significant for RTs $\left[F(1,59)=8.08, M S_{\mathrm{e}}=5,511, p<.01, \eta^{2}=.12\right]$ and for error rates $\left[F(1,59)=6.69, M S_{\mathrm{e}}=.004, p<.05, \eta^{2}=\right.$ .10] . Participants responded more slowly and less accurately to targets that were preceded by a trial in a different modality than to targets that were preceded by a trial in the same modality. This effect did not interact with modality (both $F_{S}<1$ ).

Next, we correlated each person's scores for the QMI, OSIQ, and mental rotation slope with their overall modality-switch effects. ${ }^{2}$ The results of these correlations are displayed in Table 3. None of the imagery measures showed a significant correlation with the overall modalityswitch effects (all $p s>.10)$. This lack of correlation might

Table 4

Correlations Among Scores for the Modality-Switch Effects, Questionnaire on Mental Imagery (QMI), Object-Spatial Imagery Questionnaire (OSIQ), and Rotation Slope

\begin{tabular}{|c|c|c|c|c|c|c|c|c|c|c|c|c|c|c|c|c|}
\hline Measure & 1 & 2 & 3 & 4 & 5 & 6 & 7 & 8 & 9 & 10 & 11 & 12 & 13 & 14 & 15 & 16 \\
\hline \multicolumn{17}{|l|}{ Modality Switch RT } \\
\hline 1. Auditory & - & & & & & & & & & & & & & & & \\
\hline 2. Tactile & .06 & - & & & & & & & & & & & & & & \\
\hline 3. Visual accuracy & -.13 & $-.40^{* *}$ & - & & & & & & & & & & & & & \\
\hline 4. Auditory & .01 & .03 & -.06 & - & & & & & & & & & & & & \\
\hline 5. Tactile & .19 & -.16 & -.09 & -.20 & - & & & & & & & & & & & \\
\hline 6. Visual & -.05 & .10 & .12 & -.06 & -.03 & - & & & & & & & & & & \\
\hline \multicolumn{17}{|l|}{ QMI } \\
\hline 7. Auditory & -.03 & -.15 & -.05 & -.03 & .09 & .13 & - & & & & & & & & & \\
\hline 8. Tactile & -.03 & -.23 & -.03 & .07 & -.14 & -.15 & $.51^{* *}$ & - & & & & & & & & \\
\hline 9. Visual & -.24 & -.17 & .23 & .06 & .05 & .04 & $.28^{*}$ & .06 & - & & & & & & & \\
\hline 10. Gustatory & .01 & -.11 & -.04 & .05 & .02 & .04 & $.70^{* *}$ & $.58^{* *}$ & $.31^{*}$ & - & & & & & & \\
\hline 11. Olfactory & .04 & -.14 & .03 & .16 & .02 & .13 & $.67^{* *}$ & $.42^{* *}$ & $.30^{*}$ & $.57^{* *}$ & - & & & & & \\
\hline 12. Kinaesthetic & .01 & -.10 & .11 & -.25 & -.11 & .01 & $.40^{* *}$ & $.59^{* *}$ & -.04 & $.39^{* *}$ & $.38^{* *}$ & - & & & & \\
\hline 13. Organic & .02 & -.11 & .09 & $-.31^{*}$ & -.01 & -.06 & .24 & $.32^{*}$ & -.03 & .19 & .11 & $.40^{* *}$ & - & & & \\
\hline \multicolumn{17}{|l|}{ OSIQ } \\
\hline 14. Object & .18 & .16 & -.03 & .12 & .00 & .13 & -.22 & -.16 & $-.52^{* *}$ & -.23 & -.22 & -.10 & -.06 & - & & \\
\hline 15. Spatial & -.10 & .06 & .03 & $-.30^{*}$ & .07 & -.11 & .01 & .02 & .11 & .03 & -.21 & .04 & .15 & -.21 & - & \\
\hline \multicolumn{17}{|l|}{ Rotation } \\
\hline 16. Slope & -.10 & -.05 & .08 & .07 & .00 & .23 & .03 & -.20 & $.33^{*}$ & -.02 & .18 & -.15 & -.24 & .01 & -.15 & - \\
\hline
\end{tabular}


be due to pooling all modalities. Therefore, we separated all scores by modality and did another correlation analysis, displayed in Table 4. First, consider the relation between vividness and modality switch. Of the 42 correlations (7 QMI subscales $\times 3$ modalities $\times 2$ measures), only the correlation between the accuracy effect for auditory properties and vividness for organic imagery was significant. This was likely due to chance. Thus, there is no systematic relation between vividness of imagery and modality switch.

The modality-switch effect was also not related to people's preference for and experience with object-oriented and spatial imagery. Of the 12 correlations, only the correlation between the accuracy effect for auditory properties and spatial imagery was significant. Again, this was likely due to chance. There were also no significant correlations between mental rotation and the modality-switch effect.

Finally, we investigated the relations among the various measures of imagery. There were many significant correlations between the subscales of the QMI. Except for the vividness of visual and organic imagery, imagery in all other modalities was highly intercorrelated. Vividness of visual imagery was correlated with object imagery and mental rotation performance. These findings are important, because they show that the vividness scale did measure both imagery preference and imagery ability, and our data have enough power to detect such correlations.

\section{DISCUSSION}

In the present study, we investigated the relationship between imagery and the modality-switch effect in a property verification task. That we found no systematic relation suggests that the perceptual simulations that underlie conceptual representations are fundamentally different from conscious mental imagery. If they were based on the same mechanisms, we would expect larger modalityswitch effects with higher ratings of reported vividness. We also would expect larger modality-switch effects with higher ratings of reported object imagery. Finally, we would expect larger modality-switch effects with larger mental rotation slopes. Because none of these relations were found, we conclude that perceptual simulation and imagery ability are not the same.

Imagery may not be a single construct but may rather consist of a collection of processes that can be differentiated (Blajenkova et al., 2006; Burton \& Fogarty, 2003; Kosslyn et al.,1984; Kozhevnikov et al., 2005). In the present study, we have tried to use measures that together cover a wide range of these components. With the two self-report questionnaires, we measured the ability to use and preference for using imagery for seven different modalities and for object versus spatial processing. With the mental rotation task, we measured the ability to generate, manipulate, and compare visual mental images. If imagery underlies conceptual representation, all these factors should be related to the processes that are involved in the property verification task and in modality switching. Of course, it is possible that we missed other components of imagery and that these components underlie simulation.
Our results suggest, however, that imagery and simulation do not share much processing.

An important difference between simulation and imagery might be that simulations happen automatically and that only the end products reach awareness. In contrast, imagery consists of a set of effortful processes that can be used strategically to perform a task. In fact, in most imagery research, participants are explicitly instructed to inspect or manipulate a mental image. For example, Kosslyn et al. (1984) had participants study two line gratings, close their eyes, imagine the gratings moving apart until the lines blurred, and then indicate the distance between the two gratings in their mental image. Individual differences in imagery might reflect participants' ability to follow these instructions, perform each operation, and stay focused on the mental image. It is possible that the simulations that underlie conceptual representation are also used during imagery, but that the individual differences in imagery are largely due to the more effortful conscious processes. Thus, simulation may be one of the components of imagery, but, because simulation is automatic, it contributes little variability to overall performance.

In conclusion, the present study showed that representation and imagery are not correlated. This finding suggests that the effect of perceptual manipulations on concept representations is not due to conscious imagery, and it supports the idea that perceptual simulation is central to knowledge about concepts.

\section{AUTHOR NOTE}

This research was supported by a grant to D.P. from the Netherlands Organization for Scientific Research (NWO). We thank Maaike Habra for her assistance, Art Glenberg, Olivier Corneille, and an anonymous reviewer for their constructive comments, and René Zeelenberg for helpful discussions. Address correspondence to D. Pecher, Psychology Department, T12-33, Erasmus University Rotterdam, Postbus 1738, 3000 DR Rotterdam, The Netherlands (e-mail: pecher@fsw.eur.nl).

\section{REFERENCES}

Barsalou, L. W. (1999). Perceptual symbol systems. Behavioral \& Brain Sciences, 22, 577-660.

Barsalou, L. W. (2008). Grounded cognition. Annual Review of Psychology, 59, 617-645.

Barsalou, L. W. (2009). Simulation, situated conceptualization, and prediction. Philosophical Transactions of the Royal Society B, 364, 1281-1289. doi:10.1098/rstb.2008.0319

Barsalou, L. W., Simmons, W. K., Barbey, A. K., \& Wilson, C. D. (2003). Grounding conceptual knowledge in modality-specific systems. Trends in Cognitive Sciences, 7, 84-91.

BetTs, G. H. (1909). The distribution and functions of mental imagery (Contributions to Education Series, No. 26). New York: Teachers College, Columbia University.

Blajenkova, O., Kozhevnikov, M., \& Motes, M. A. (2006). Objectspatial imagery: A new self-report imagery questionnaire. Applied Cognitive Psychology, 20, 239-263.

Borst, G., \& KossLyn, S. M. (2008). Visual mental imagery and visual perception: Structural equivalence revealed by scanning processes. Memory \& Cognition, 36, 849-862. doi:10.3758/MC.36.4.849

Burton, L. J., \& Fogarty, G. J. (2003). The factor structure of visual imagery and spatial abilities. Intelligence, 31, 289-318.

Glenberg, A. M. (1997). What memory is for. Behavioral \& Brain Sciences, 20, 1-55.

Goldberg, R. F., Perfetti, C. A., \& Schneider, W. (2006). Perceptual knowledge retrieval activates sensory brain regions. Journal of Neuroscience, 26, 4917-4921. 
Kosslyn, S. M., Brunn, J., CAVE, K. R., \& Wallach, R. W. (1984). Individual differences in mental imagery ability: A computational analysis. Cognition, 18, 195-243.

Kozhevnikov, M., Kosslyn, S., \& Shephard, J. (2005). Spatial versus object visualizers: A new characterization of visual cognitive style. Memory \& Cognition, 33, 710-726.

Laws, K. R. (2002). Category-specific naming and modality-specific imagery. Brain \& Cognition, 48, 418-420.

Mahon, B. Z., \& Caramazza, A. (2008). A critical look the embodied cognition hypothesis and a new proposal for grounding conceptual content. Journal of Physiology, 102, 59-70.

MARKs, D. F. (1973). Visual imagery differences in the recall of pictures. British Journal of Psychology, 64, 17-24.

Marques, J. F. (2006). Specialization and semantic organization: Evidence for multiple semantics linked to sensory modalities. Memory \& Cognition, 34, 60-67.

Martin, A., Haxby, J. V., Lalonde, F. M., Wiggs, C. L., \& UngerLEIDER, L. G. (1995). Discrete cortical regions associated with knowledge of color and knowledge of action. Science, 270, 102-105.

Pecher, D., Zeelenberg, R., \& Barsalou, L. W. (2003). Verifying different-modality properties for concepts produces switching costs. Psychological Science, 14, 119-124.

Pecher, D., Zeelenberg, R., \& Barsalou, L. W. (2004). Sensorimotor simulations underlie conceptual representations: Modality-specific effects of prior activation. Psychonomic Bulletin \& Review, 11, 164167.

Pulvermüller, F. (1999). Words in the brain's language. Behavioral \& Brain Sciences, 22, 253-336.

Rouw, R., Kosslyn, S. M., \& Hamel, R. (1997). Detecting high-level and low-level properties in visual images and visual percepts. Cognition, 63, 209-226.

SheEhan, P. W. (1967). A shortened form of Betts' Questionnaire upon mental imagery. Journal of Clinical Psychology, 23, 386-389.

Solomon, K. O., \& Barsalou, L. W. (2001). Representing properties locally. Cognitive Psychology, 43, 129-169.
Solomon, K. O., \& Barsalou, L. W. (2004). Perceptual simulation in property verification. Memory \& Cognition, 32, 244-259.

Spence, C., Nicholls, M. E. R., \& Driver, J. (2001). The cost of expecting events in the wrong sensory modality. Perception \& Psychophysics, 63, 330-336.

Stanfield, R. A., \& ZWAan, R. A. (2001). The effect of implied orientation derived from verbal context on picture recognition. Psychological Science, 12, 153-156.

Van Dantzig, S., \& Pecher, D. (2009). Norms for the modalityexclusivity of 392 concept-property items. Unpublished manuscript.

van Dantzig, S., Pecher, D., Zeelenberg, R., \& Barsalou, L. W. (2008). Perceptual processing affects conceptual processing. Cognitive Science, 32, 579-590.

Vermeulen, N., Corneille, O., \& Niedenthal, P. M. (2008). Sensory load incurs conceptual processing costs. Cognition, 109, 287-294.

Vermeulen, N., Niedenthal, P. M., \& Luminet, O. (2007). Switching between sensory and affective systems incurs processing costs. Cognitive Science, 31, 183-192.

\section{NOTES}

1. Some obsolete or culturally specific items were replaced by a different item from Betts's original questionnaire or by a new item. The following old-new items were replaced: (auditory) The whistle of a locomotive-The chinking of glasses striking together and The sound of escaping steam - The rustling of a newspaper; (tactile) A fur muff-A smooth doorknob; and (olfactory) cooking cabbage-sautéed onions. Two gustatory items were replaced to have a better distribution of tastes: oranges-grapefruit and jelly-yoghurt.

2 . Analyses with the residuals of the regressions of the switch on the no-switch means yielded a similar pattern of results.

(Manuscript received April 6, 2009; revision accepted for publication June 9, 2009.) 